\title{
Involvement of mitochondrial dynamics in the antineoplastic activity of cisplatin in murine leukemia L1210 cells
}

\author{
XIAO-JIAN HAN ${ }^{1,2^{*}}$, SHENG-LAN SHI ${ }^{2,3^{*}}$, YONG-FANG WEI ${ }^{3}$, LI-PING JIANG ${ }^{3}$, \\ MIAO-YU GUO ${ }^{2}$, HONG-LI WU ${ }^{1}$ and YU-YING WAN ${ }^{1}$ \\ ${ }^{1}$ Department of Intra-Hospital Infection Management, The Second Affiliated Hospital of Nanchang University; \\ ${ }^{2}$ Research Institute of Ophthalmology and Visual Sciences, Affiliated Eye Hospital of Nanchang University; \\ ${ }^{3}$ Department of Pharmacology, School of Pharmaceutical Sciences, Nanchang University, \\ Nanchang, Jiangxi 330006, P.R. China
}

Received October 28, 2016; Accepted May 18, 2017

DOI: 10.3892/or.2017.5765

\begin{abstract}
Leukemia is a type of hematopoietic stem cell malignant cloned disease with high mortality. Cisplatin-based chemotherapy is one of the most common treatments for leukemia. Similar to other chemotherapeutic agents, cisplatin resistance has become a serious issue in cancer therapy. In the present study, we investigated the role of mitochondrial dynamics in the antineoplastic activity of cisplatin in murine leukemia L1210 cells. Firstly, the L1210 cell line resistant to cisplatin (L1210/DDP) was established. Compared to its parental cell line, the $\mathrm{IC}_{50}$ value of cisplatin in the L1210/DDP cells was increased 10-fold. Mitofusins (Mfn1 and Mfn2), mitochondrial outer membrane fusion proteins, were markedly upregulated in the L1210/DDP cells, whereas the expression of fission protein Drp1 and inner membrane fusion protein OPA1 were not significantly altered. In addition, mitofusins were also upregulated in the parental L1210 cells subjected to cisplatin stress. To investigate the role of mitochondrial dynamics in the antineoplastic activity of cisplatin, the effect of mitochondrial division inhibitor (Mdivi)-1 on cisplatin-induced cell death, caspase- 3 cleavage and ROS production was examined in L1210 cells. We found that $5 \mu \mathrm{M}$ of Mdivi-1 efficiently attenuated cisplatin-induced cell death, caspase activation and intracellular ROS increase in L1210 cells. Our data indicated that mitochondrial dynamics play an important role in the antineoplastic activity of cisplatin, and mitofusin-mediated mitochondrial fusion may be involved in the process of
\end{abstract}

Correspondence to: $\mathrm{Dr}$ Yu-Ying Wan, Department of Intra-Hospital Infection Management, The Second Affiliated Hospital of Nanchang University, 1 Minde Road, Donghu, Nanchang, Jiangxi 330006, P.R. China

E-mail:wanyy79@hotmail.com

*Contributed equally

Key words: leukemia, cisplatin resistance, L1210 cells, mitochondrial dynamics, apoptosis cisplatin resistance in leukemia cells. Therefore, the present study revealed that mitochondrial dynamics may be a potential target used to improve the antineoplastic activity of cisplatin in leukemia in the future.

\section{Introduction}

Leukemia is also known as blood cancer and has a high mortality rate. It is pathologically characterized by the massive proliferation of abnormal hematopoietic stem cells $(1,2)$. Although the precise pathogenesis of leukemia remains to be elucidated, various genetic mutations occurring in response to factors including genetic factors, immune deficiency, toxic agents and the leukosis virus in the process of leukocyte differentiation have been widely accepted as the main causes of leukemia (3). Genetic mutations may lead to the unlimited proliferation of leukemia cells in bone marrow and other hematopoietic tissue (4). As a result, the development of mature blood cells is disrupted. Due to the high mortality rate, leukemia is considered as a malignant cancer which seriously threatens human health. It has also been reported that the incidence rate of leukemia is relatively high in the elderly population and children in China (5). At present, the main treatment methods for leukemia are combined chemotherapy and marrow transplantation. However, the high expense, donor deficiency and immunological rejection are important issues in marrow transplantation (6-8). Thus, combined chemotherapy is the most important treatment for leukemia. However, among conventional chemotherapeutic agents, cisplatin is also widely used in combined chemotherapy for leukemia (9). Unfortunately, evidence has shown that with treatment more and more cancer cells become resistant to cisplatin (10). Therefore, it is urgent to further investigate the precise mechanism of cisplatin resistance.

Platinum-based drugs are widely used in the treatment of cancer such as leukemia, lymphomas, melanoma, head-neck cancer, bladder cancer and gynecological tumors (11). Cisplatin is one of the first platinum-based drugs discovered in the 1960s (12). Cisplatin interacts with DNA double strands by forming interstrand and intrastrand adducts, thereby inducing apoptosis in cancer cells through the interference with DNA 
replication and gene transcription (13). Similar to other chemotherapeutic agents, the effect of cisplatin is commonly limited by the resistance of cancer cells. Cisplatin resistance can be intrinsic or acquired. Intrinsic resistance means that cancer cells retain certain featured gene expression profiles contributing to resistance prior to cisplatin treatment. In contrast, acquired resistance occurs in cancer cells after cisplatin-induced epigenetic modulation and gene mutation (13). In clinical treatment, cisplatin often results in the development of chemoresistance, despite a consistent rate of initial responses. Acquired cisplatin resistance is also the most common cause of therapeutic failure, and leads to leukemia recurrence.

A primary study revealed that cisplatin-induced cell death was mostly caused by nuclear DNA damage (14). However, it was recently discovered that mitochondial DNA, or other mitochondrial targets may be more important than nuclear DNA in cisplatin-induced cell death (15). Mitochondria are well known for their essential function in the production of ATP. In fact, mitochondria are involved in a variety of cellular processes, including survival, proliferation and apoptosis (16-18). Mitochondria are also highly dynamic organelles and move through the cell with frequent fission and fusion events (19). Various highly conserved dynamin-related GTPases are identified as the mediators of mitochondrial dynamics. Dynamin-related protein 1 (Drpl) is involved in the process of mitochondrial fission, while mitofusin $1 / 2(\mathrm{Mfn} 1 / 2)$ and OPA1 are required for mitochondrial outer or inner membrane fusion in mammalian cells, respectively (20). In addition, recent studies have suggested the involvement of mitochondrial dynamics in the acquired cisplatin resistance or sensitivity in several cancer cell lines $(21,22)$. It has been reported that OPA-1-mediated mitochondrial fusion is potentially responsible for cisplatin-induced resistance in neuroblastoma B50 rat cells (21). By contrast, Drp1-dependent mitochondrial fission was found to regulate piceatannol-induced cisplatin sensitivity in ovarian cancer (22). Therefore, it is of interest to investigate the role of mitochondrial dynamics in the antineoplastic activity of cisplatin in leukemia cells.

In the present study, we established the L1210/DDP cell line, and found that the $\mathrm{IC}_{50}$ value of cisplatin in the L1210/DDP cells was increased 10-fold. In addition, mitochondrial outer membrane fusion proteins, Mfn1 and Mfn2 were upregulated in L1210/DDP cells. In addition, mitofusins were also upregulated in the parental L1210 cells subjected to cisplatin stress. The Drp1 inhibitor, Mdivi-1, efficiently attenuated cisplatin-induced cell death, caspase activation and intracellular ROS increase in L1210 cells. Our data indicate that mitofusins and Drpl-mediated mitochondrial dynamics may be involved in the antineoplastic activity of cisplatin in L1210 cells, and suggest that mitochondria may be potential targets used to improve the clinical outcomes of leukemia in the future.

\section{Materials and methods}

Cell culture. Leukemia cell line L1210 was obtained from the China Center for Type Culture Collection (CCTCC; Wuhan, China). The L1210/DDP cell line was generated according to the dose-escalation strategy as previously described $(23,24)$. In brief, the parental L1210 cells were treated with cisplatin at an $\mathrm{IC}_{90}$ concentration, and the surviving cells were cloned in soft agar. A clone was selected and cultured in medium supplemented with a first dose of $0.4 \mathrm{mg} / \mathrm{l}$ of cisplatin (Sigma-Aldrich, St. Louis, MO, USA). The dose of cisplatin in the medium for the L1210 cells was increased 50\% at every time-point of subculture. The surviving cells followed by six subcultures were cloned again in soft agar. A clone was selected as the cisplatin-resistant cell line for culture in the medium. The cells were grown in suspension in medium (Gibco, Grand Island, NY, USA) supplemented with $10 \%$ fetal bovine serum (FBS; TransGen Biotech, Beijing, China) and 1\% penicillin/streptomycin (P/S) (Solarbio, Beijing, China), and maintained in a humidified incubator (Thermo Fisher Scientific, Waltham, $\mathrm{MA}, \mathrm{USA}$ ) at $37^{\circ} \mathrm{C}$ with an atmosphere containing $5 \% \mathrm{CO}_{2}$.

Cell viability assay. Cell viability was determined by trypan blue exclusion assay as previously described (25). In brief, L1210 and L1210/DDP cells were firstly seeded at $5 \times 10^{5}$ in 24-well plates and maintained in suspension culture. At $72 \mathrm{~h}$ after incubation with DDP or Mdivi-1 (Sigma-Aldrich) + DDP, samples were centrifuged at $1,000 \mathrm{xg}$ for $5 \mathrm{~min}$ at $25^{\circ} \mathrm{C}$. The cells were stained with $0.04 \%$ trypan blue (Sigma-Aldrich) after being washed with phosphate-buffered saline (PBS). The number of dead cells (blue) and viable cells (uncolored) were counted using a hemacytometer. The ratio of the number of dead cells/all counted cells represented the percentage of cell death. The $\mathrm{IC}_{50}$ value of cisplatin to L1210 or L1210/DDP was determined in the same way.

Western blot analysis. L1210 and L1210/DDP cells were harvested and lyzed using radioimmunoprecipitation assay (RIPA) lysis buffer (Solarbio) according to the manufacturer's instructions. Whole cell lysates were mixed with an equal volume of $2 \mathrm{X}$ loading buffer (25\% glycerol, $2 \%$ sodium dodecyl sulfate, $5 \% \beta$-mercaptoethanol, $0.01 \%$ bromophenol blue and $1 \mathrm{M}$ Tris- $\mathrm{HCl}$ ), sonicated, boiled for $5 \mathrm{~min}$ and stored at $-20^{\circ} \mathrm{C}$ prior to use. The cell lysates were subjected to sodium dodecyl sulphate-polyacrylamide gel electrophoresis (SDS-PAGE). After electrophoresis, the proteins were transferred onto polyvinylidene difluoride (PVDF) membranes (Millipore, Billerica, MA, USA). The membranes were blocked with 5\% skim milk in Tris-buffered saline with Tween-20 (TBST) buffer for $1 \mathrm{~h}$ at room temperature, and then, immunoblotted for $2 \mathrm{~h}$ at room temperature with the following primary antibodies: rabbit anti-Drp1, Mfn2 and caspase-3 (1:1,000; Cell Signaling, Boston, MA, USA), rabbit anti-Mfn1 and OPA-1 (1:1,000; Abcam, Cambridge, UK), and rabbit anti-GAPDH (1:1,000; Santa Cruz Biotechnology, Inc., Dallas, TX, USA). After three washes with TBST, the membranes were further incubated with an HRP-conjugated goat anti-rabbit secondary antibody (1:2,000; TransGen Biotech) for $2 \mathrm{~h}$ at room temperature. A chemiluminescence assay was carried out with Amersham ECL Prime Western Blotting Detection reagents (CWBIO, Beijing, China), and the immunoblotting signal was detected using Molecular Imager ${ }^{\circledR}$ ChemiDoc $^{\mathrm{TM}}$ XRS+ system (Bio-Rad, Hercules, CA, USA).

Annexin V-FITC/PI apoptosis assay. Subsequent to the indicated treatments, the cells were harvested from each group for the apoptosis assay using Annexin V-fluorescein isothiocyanate (Annexin V-FITC) and propidium iodide (PI) 
Table I. Cisplatin cytotoxicity in the L1210 cells.

\begin{tabular}{lcccccccccccc}
\hline & \multicolumn{10}{c}{ Cisplatin concentration in the L1210 cells (mg/l) } \\
\cline { 2 - 11 } & 0 & 0.025 & 0.05 & 0.1 & 0.2 & 0.4 & 0.8 & 1.2 & 1.6 & 2 & 3.2 & 6.4 \\
\hline Percentage of cell death (\%) & 13.43 & 23.77 & 27.46 & 28.18 & 30.70 & 39.53 & 49.28 & 53.23 & 65.30 & 73.25 & 86.78 & 90.57 \\
\hline
\end{tabular}

L1210 cells were exposed to different concentrations of cisplatin. At $72 \mathrm{~h}$ after cisplatin treatment, the cell viability was examined by trypan blue exclusion assay, and the percentage of cell death was calculated.

Table II. Cisplatin cytotoxicity in the L1210/DDP cells.

\begin{tabular}{lccccccccccc}
\hline & \multicolumn{8}{c}{ Cisplatin concentration in the L1210/DDP cells (mg/l) } \\
\cline { 2 - 11 } & 0 & 0.25 & 0.5 & 1 & 2 & 4 & 8 & 16 & 32 & 64 \\
\hline Percentage of cell death (\%) & 9.84 & 13.06 & 15.84 & 16.73 & 26.41 & 44.44 & 51.89 & 74.48 & 60.74 & 93.85
\end{tabular}

L1210/DDP cells were exposed to different concentrations of cisplatin. At $72 \mathrm{~h}$ after cisplatin treatment, the cell viability was examined by trypan blue exclusion assay, and the percentage of cell death was calculated.

A

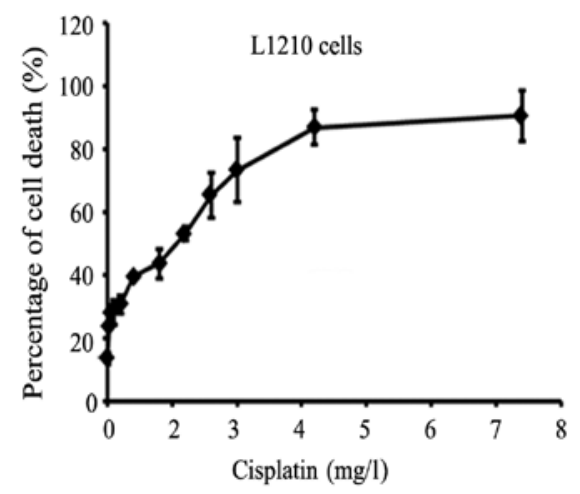

B

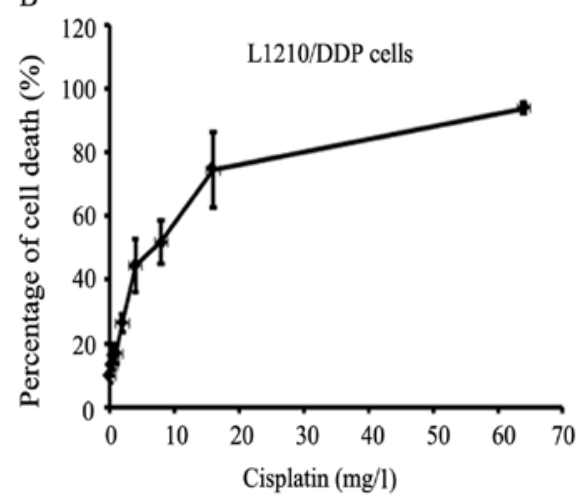

Figure 1. The dose-response curves of L1210 and L1210/DDP cells to cisplatin. (A) The dose-response curve of L1210 cells to cisplatin. (B) The dose-response curve of L1210/DDP cells to cisplatin. The percentage of cell death in the L1210 and L1210/DDP cells after treatment with different concentrations of cisplatin, was obtained from at least three independent experiments. Then, the dose-response curves of the two cell lines to cisplatin were generated, and the $\mathrm{IC}_{50}$ values for cisplatin in the two cell lines were also calculated, respectively.

(4A Biotech Co., Ltd., Beijing, China) double staining. The cells were resuspended in $100 \mu \mathrm{l}$ of binding buffer with $5 \mu 1$ of Annexin V-FITC and $200 \mathrm{ng}$ of PI, and incubated for $15 \mathrm{~min}$ at room temperature in the dark. Then, the samples were subjected to the apoptosis assay using flow cytometry, and the data were processed using the Guawa Nexin software (Guava, Millipore Corp.).

Detection of the intracellular ROS level. To examine the role of Drp1-dependent mitochondrial fission in intracellular ROS production, cells were pretreated with $5 \mu \mathrm{M}$ Mdivi-1 for $2 \mathrm{~h}$ prior to CDDP treatment. At $72 \mathrm{~h}$ after cisplatin treatment, the cells were incubated with $10 \mu \mathrm{M}$ of the fluorescent probe 2',7'-dichlorodihydrofluorescein diacetate (DCFH-DA; Sigma-Aldrich) for $30 \mathrm{~min}$ at $37^{\circ} \mathrm{C}$ in the dark. After incubation, the cells were washed twice with PBS and harvested. The fluorescence intensity was assessed using flow cytometry with the excitation source at $488 \mathrm{~nm}$ and the emission wavelength at $525 \mathrm{~nm}$, thus detecting the intracellular ROS. Data analysis was carried out using inCyte software (both from Guava, Millipore Corp.).

Statistical analysis. The quantitative data are displayed as the mean \pm SD. Data were analyzed using either Student's t-test to compare two conditions or ANOVA followed by planned comparisons of multiple conditions, and $\mathrm{p}<0.05$ was considered to indicate a statistically significant result.

\section{Results}

Establishment of the L1210/DDP cell line and confirmation of its cisplatin resistance. In order to investigate the 

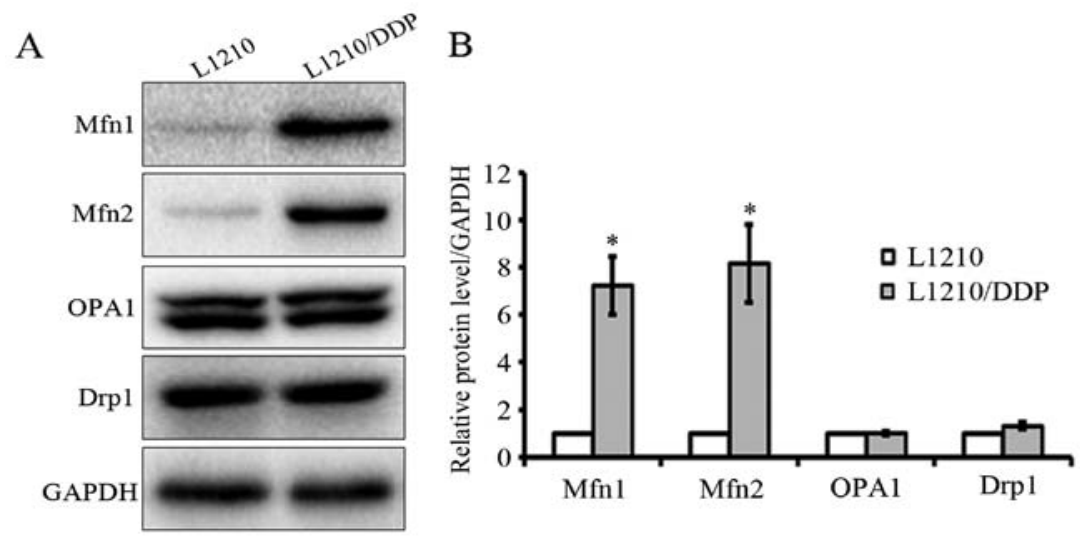

Figure 2. Expression level of mitochondrial dynamic-related proteins in L1210 and L1210/DDP cell lines. (A) The expression of mitochondrial dynamicrelated proteins in the two cell lines was examined using western blot assay. GAPDH was used as an endogenous reference. (B) Quantification of the relative expression levels of mitochondrial dynamic-related proteins (MRPs) is indicated as the normalization of the ratio of MRP/GAPDH in each sample. Data are presented as the mean \pm SD of at least three independent experiments; ${ }^{*} \mathrm{p}<0.05$ vs. the L1210 cells.

mechanism of cisplatin resistance, we successfully generated the L1210/DDP cell line according to a dose-escalation strategy $(23,24)$. The parental cells were grown in suspension in RPMI-1640 medium supplemented with $10 \%$ FBS and $1 \%$ $\mathrm{P} / \mathrm{S}$, whereas the L1210/DDP cells were maintained in medium containing $4 \mathrm{mg} / \mathrm{l}$ of cisplatin to retain their resistance. The cisplatin resistance of the L1210/DDP cells was determined by trypan blue exclusion assay. As shown in Fig. 1A and Table I, the parental L1210 cells were sensitive to $0.8-6.4 \mathrm{mg} / \mathrm{l}$ of cisplatin. In contrast, the L1210/DDP cells were sensitive to 4-64 mg/l of cisplatin (Fig. 1B and Table II). In addition, the dose-response curves of the two cell lines to cisplatin were generated (Fig. 1A and B), and the $\mathrm{IC}_{50}$ values for cisplatin were also calculated as 0.795 and $8.131 \mathrm{mg} / \mathrm{l}$ in the L1210 and L1210/DDP cell lines, respectively. Compared to the parental cell line, the $\mathrm{IC}_{50}$ value for cisplatin in the L1210/DDP cells was increased 10 -fold.

Expression ofmitochondrialdynamic-relatedproteins in $L 1210$ and L1210/DDP cells. It has been reported that mitochondrial dynamics are involved in acquired cisplatin resistance (7). To investigate the possible role of mitochondrial dynamics in the cisplatin resistance of leukemia cells, the expression level of mitochondrial dynamic-related proteins was examined in the two cell lines using western blot assay. Notably, we found that both Mfn1 and Mfn2 were upregulated in L1210/DDP cells. However, there was no significant difference in the expression level of Drp1 and OPA1 in the two cell lines (Fig. 2). Since both Mfn1 and Mfn2 are important components required for mitochondrial outer membrane fusion, the results revealed the possibility that mitofusin-mediated mitochondrial fusion may contribute to the mechanism of cisplatin resistance in leukemia cells.

Effect of cisplatin stress on mitochondrial dynamic-related protein expression in the parental L1210 cells. Although mitofusins were upregulated in the L1210/DDP cells, direct evidence that mitofusin-mediated mitochondrial fusion leads to the development of cisplatin resistance in leukemia cells remains unclear. Therefore, to further confirm the involvement of mitofusins in the development of cisplatin resistance

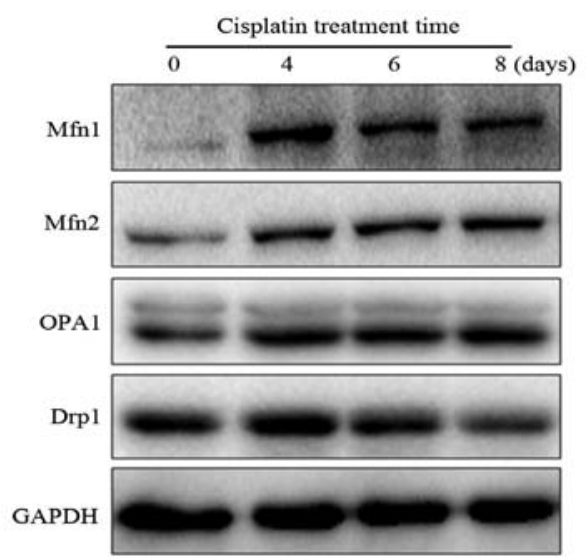

Figure 3. Expression of mitochondrial dynamic-related proteins in L1210 cells after cisplatin stress. After exposure to $0.4 \mathrm{mg} / 1$ of cisplatin for 0,4 , 6 and 8 days, the cells were harvested. Whole lysates were subjected to $8 \%$ SDS-PAGE and immunoblotted with the related antibody. GAPDH was used as an endogenous reference.

in leukemia cells, cisplatin stress at $0.4 \mathrm{mg} / \mathrm{l}$ (the concentration below the $\mathrm{IC}_{50}$ value) was introduced to the parental L1210 cells. The expression of mitochondrial dynamic-related proteins in cells was examined at $0,4,6$ and 8 days after treatment with $0.4 \mathrm{mg} / 1$ of cisplatin. As shown in Fig. 3, it was found that both Mfn1 and Mfn2 were obviously upregulated during the period of cisplatin stress, whereas the expression of OPA1 was not significantly altered. In contrast, Drp1 was downregulated at 8 days after cisplatin stress. Although there were a few differences in the expression patterns of mitochondrial dynamic-related proteins, the results revealed that the shift of mitochondrial dynamics to fusion may contribute to the development of cisplatin resistance in L1210 cells.

Drpl inhibitor Mdivi-1 efficiently attenuates cisplatin-induced cell death in L1210 cells. The aforementioned data revealed that mitochondrial fusion may contribute to the antineoplastic activity of cisplatin in leukemia cells. To further investigate the role of mitochondrial dynamics in the sensitivity of leukemia cells to cisplatin, we used different concentrations of Mdivi-1, a Drp1 inhibitor, to decrease Drp1-dependent mitochondrial 
A

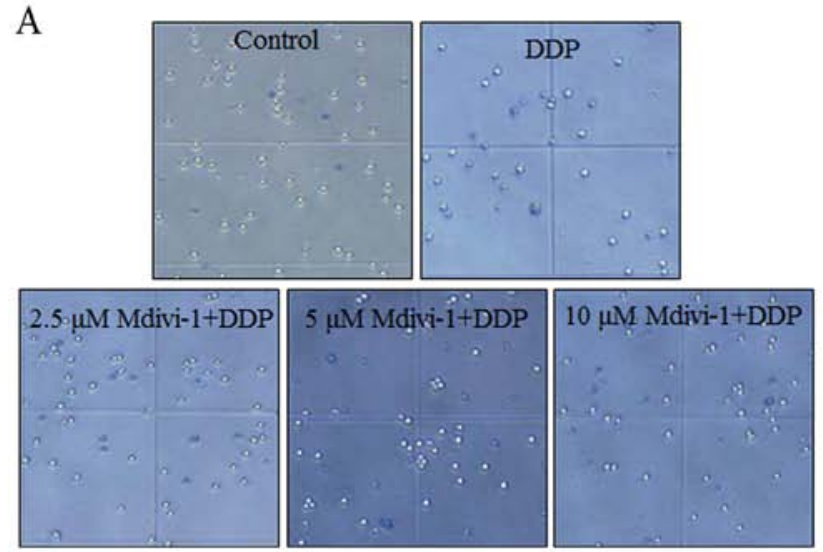

B

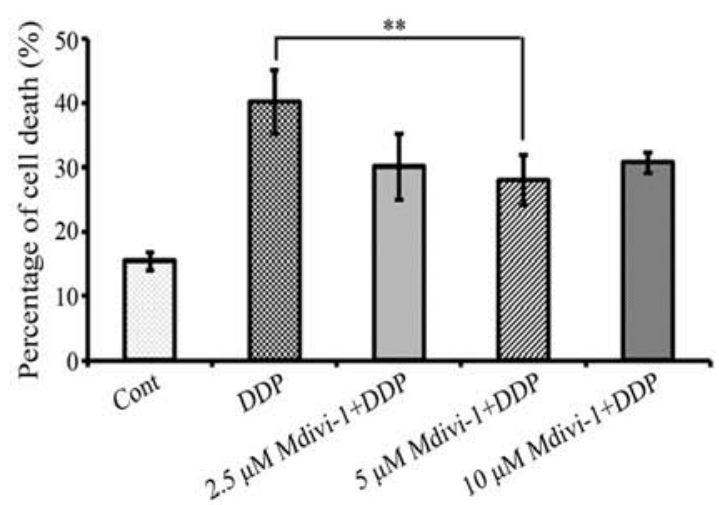

Figure 4. Effect of mitochondrial division inhibitor (Mdivi)-1 on cisplatin cytotoxicity in L1210 cells. (A) The cell viability was examined by trypan blue exclusion assay. L1210 cells were divided into five groups: control, DDP, $2.5 \mu \mathrm{M}$ Mdivi-1 + DDP, $5 \mu \mathrm{M}$ Mdivi-1 + DDP and $10 \mu \mathrm{M}$ Mdivi-1 + DDP. The representive image in each group showed the cell death (in blue) after cisplatin exposure. (B) Effect of Mdivi-1 on cisplatin cytotoxicity in L1210 cells. The ratio of the number of dead cells/all counted cells represents the percentage of cell death in each group. Data are presented as the mean \pm SD of at least three independent experiments; ${ }^{* *} \mathrm{p}<0.01$.

A

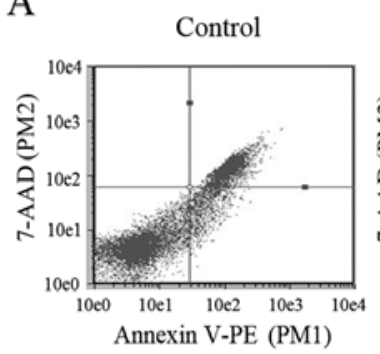

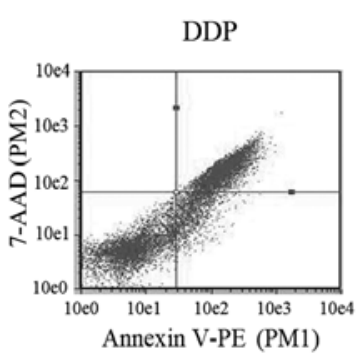

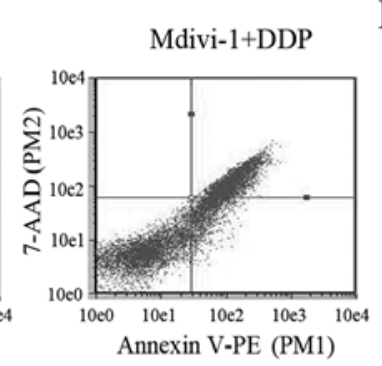

B

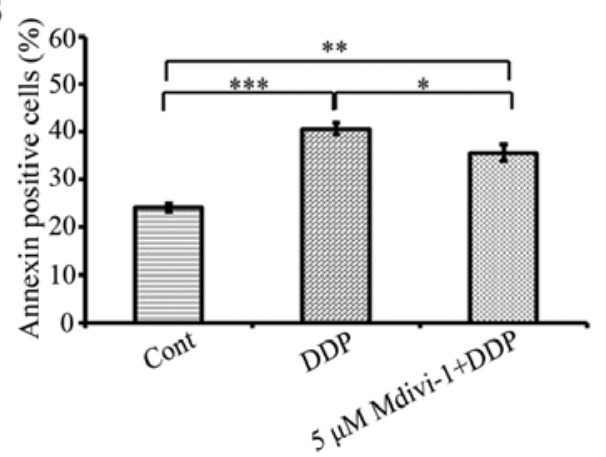

Figure 5. Mitochondrial division inhibitor (Mdivi)-1 protects L1210 cells against cisplatin cytotoxicity. (A) L1210 cells were divided into three groups: Control, DDP and $5 \mu \mathrm{M}$ Mdivi-1 + DDP. After the indicated treatments, the percentage of apoptosis in each group was examined by flow cytometry using Annexin V-FITC/PI double staining. (B) The quantification of the percentage of apoptosis in each group was performed. Data are presented as the mean \pm SD of three independent experiments; ${ }^{*} \mathrm{p}<0.05,{ }^{* *} \mathrm{p}<0.01,{ }^{* * *} \mathrm{p}<0.001$.

fission, and examined the effect of Mdivi-1 on cisplatininduced cell death in L1210 cells. In the trypan blue exclusion assay, the percentage of cell death induced by $0.4 \mathrm{mg} / \mathrm{l}$ of cisplatin was $\sim 40 \%$. Mdivi-1 $(5 \mu \mathrm{M})$ significantly attenuated $0.4 \mathrm{mg} / \mathrm{l}$ of cisplatin-induced cell death in L1210 cells, with the exception of 2.5 and $10 \mu \mathrm{M}$ of Mdivi-1 (Fig. 4). In the Annexin V-FITC/PI apoptosis assay, it was also revealed that $5 \mu \mathrm{M}$ Mdivi-1 significantly inhibited cisplatin-induced cell death in L1210 cells (Fig. 5). The results demonstrated that inhibition of mitochondrial fission contributes to the tolerance of leukemia cells to cisplatin.

Effects of Mdivi-1 on cisplatin-induced ROS production and caspase activation in L1210 cells. It has been reported that cisplatin induces cancer cell death through the promotion of intracellular ROS production and caspase activation $(26,27)$. The aforementioned data revealed that mitofusins were upregulated in L1210/DDP cells, and that the Drp1 inhibitor, Mdivi-1 attenuated cisplatin-induced L1210 cell death. It was necessary to further investigate the mechanism of mitochondrial dynamics in cisplatin-induced cell death in
L1210 cells. Thus, the effect of Mdivi-1 on cisplatin-induced ROS production and caspase activation was examined in L1210 cells. Consistent with previous studies $(26,27), 4 \mathrm{mg} / \mathrm{l}$ of cisplatin significantly increased the intracellular ROS level in L1210 cells. Pretreatment with $5 \mu \mathrm{M}$ of Mdivi- 1 significantly attenuated cisplatin-induced intracellular ROS production in L1210 cells, although it did not completely block the increment of ROS (Fig. 6). Moreover, the results of western blotting revealed that $4 \mathrm{mg} / \mathrm{l}$ of cisplatin markedly stimulated the cleavage of caspase-3. Pretreatment with $5 \mu \mathrm{M}$ of Mdivi-1 attenuated the cleavage of caspase-3 in L1210 cells (Fig. 7). These data demonstrated that mitochondrial dynamics may be involved in cisplatin-induced L1210 cell death through the regulation of intracellular ROS production and the caspase pathway.

\section{Discussion}

Leukemia is the most common hematopoietic malignancy. The development and progression of leukemia is a multifactorial and multi-step process that involves genetic and 
A
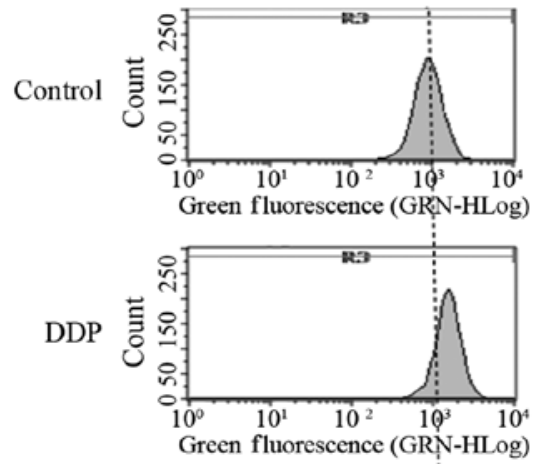

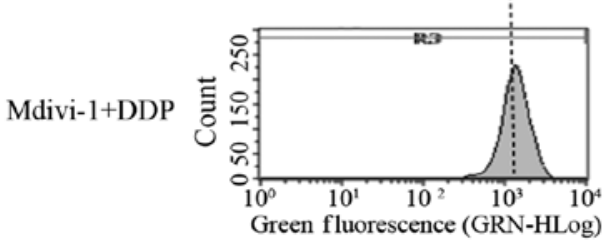

B

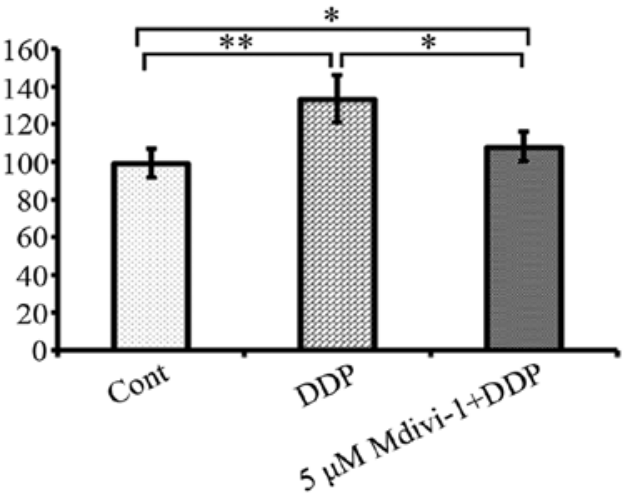

Figure 6. Effect of mitochondrial division inhibitor (Mdivi)-1 on cisplatin-induced intracellular ROS production in L1210 cells. (A) L1210 cells were divided into three groups: control, DDP and $5 \mu \mathrm{M}$ Mdivi-1 + DDP. After the indicated treatments, the intracellular ROS level in cells was examined by flow cytometry using DCFH-DA staining. (B) The relative ROS level was quantified in each group. Data are presented as the mean \pm SD of at least three independent experiments; ${ }^{*} \mathrm{p}<0.05,{ }^{* *} \mathrm{p}<0.01$.

epigenetic changes (28). Leukemia is also one of the most common malignant tumors, particularly in children. At present, combined chemotherapy is the major treatment method for leukemia, and cisplatin is one of the most common agents used for combined chemotherapy in clinical treatment. Cisplatin was also the first platinum-based drug to obtain approval of the US Food and Drug Administration (29). The antineoplastic effects of cisplatin have been linked to its ability to crosslink with DNA, then interfere with DNA repair mechanisms, cause DNA damage and eventually lead to apoptosis in cancer cells $(14,24)$. In contrast, mitochondria are also important targets of cisplatin. Cisplatin can induce the decline of ATP enzyme activity and activate the endogenous apoptosis pathway (26). Cisplatin is currently applied for the clinical management of patients suffering from leukemia, testicular, ovarian, head and neck, colorectal and lung cancers (11,30-32). However, the resistance of cancer cells to cisplatin has become a serious problem, and greatly limits its therapeutic effect in clinical treatment.

In the present study, the role of mitochondrial dynamics in cisplatin resistance of leukemia L1210 cells was investigated. Firstly, we established the L1210/DDP cell line using the dose-escalation strategy. The tolerance of L1210/DDP cells to cisplatin was increased 10 -fold, compared to the parental cells (Fig. 1). Notably, mitofusins Mfn1 and Mfn2 were obviously upregulated in L1210/DDP cells (Fig. 2). Mfn1 and Mfn2 are vital components required for mitochondrial outer membrane fusion (33). In addition, it has been reported that mitofusins are involved in apoptosis by interacting with Bak and Bax (34-36). To examine the role of mitofusins in the development of cisplatin in L1210 cells, the expression of mitochondrial dynamic-related proteins was detected after cisplatin stress. Similarly, Mfn1 and Mfn2 were upregulated in L1210 cells subjected to cisplatin stress (Fig. 3). The results revealed the upregulation of mitofusins not as an effect, but as a cause in the development of cisplatin resistance. To clarify the role of mitofusin-mediated mitochondrial fusion in

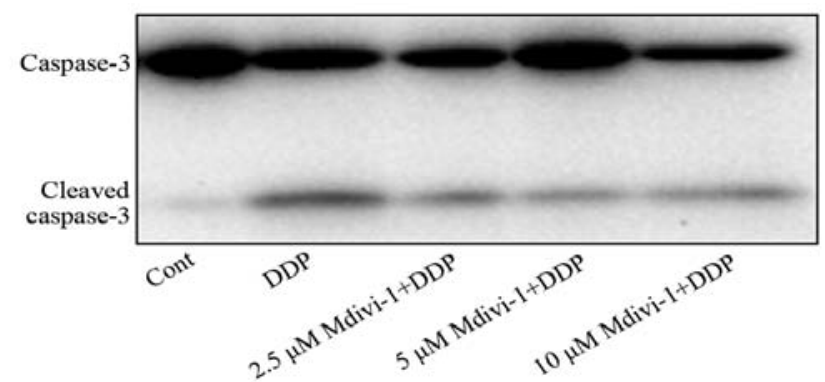

Figure 7. Mitochondrial division inhibitor (Mdivi)-1 efficiently attenuates cisplatin-induced cleavage of caspase-3 in L1210 cells. L1210 cells were divided into five groups: control, DDP, $2.5 \mu \mathrm{M}$ Mdivi-1 + DDP, $5 \mu \mathrm{M}$ Mdivi-1 + DDP and $10 \mu \mathrm{M}$ Mdivi-1 + DDP. After the indicated treatments, the cells were harvested. Whole cell lysates were prepared, subjected to SDSPAGE and immunoblotted with anti-caspase-3 antibody. The upper and lower bands indicate full length and cleaved caspase-3, respectively.

cisplatin resistance, it is necessary to knockdown mitofusins in the L1210/DDP cells or overexpress mitofusins in parental L1210 cells, and examine the antineoplastic effects of cisplatin in these cells. Unfortunately, we failed to transfect any siRNAs or plasmids into L1210 cells, and failed to infect L1210 cells with a lentivirus or adenovirus (data not shown). Thus, it is impossible to knockdown or overexpress mitofusins in L1210 cells using gene manipulation technology.

The balance between mitochondrial fission and fusion is important to maintain mitochondrial morphology. Thus, we disrupted the dynamic balance using chemical agents, to further investigate the mechanism of mitochondrial dynamics in the development of cisplatin resistance. It has been well documented that Mdivi-1 is a potent Drp1 inhibitor, and is most widely used $(37,38)$. Obvious mitochondrial fusion in cells can be observed when Drp1-dependent mitochondrial fission was inhibited by Mdivi-1 $(39,40)$. In the present study, $5 \mu \mathrm{M}$ of Mdivi-1 efficiently attenuated cisplatin-induced cell death, intracellular ROS production and caspase activation 
in L1210 cells. These results demonstrated that mitochondrial dynamics play an important role in the antineoplastic activity of cisplatin in leukemia cells. However, there are some deficiencies that warrant improvement in the present study. First, it is necessary to observe and compare mitochondrial morphology in L1210 and L1210/DDP cells, since mitofusins are upregulated in L1210/DDP cells. The two common methods used to label transfected mitochondria are Mito-DsRed and MitoTracker staining $(41,42)$. As L1210 cells are difficult to be transfected, we stained cells with MitoTracker, and found that mitochondria were clustered around the perinuclear region in the two cell lines. The two cell lines cultured in suspension appear as small spheres. As a result, it was difficult to distinguish the morphology of clustered mitochondria in the two cell lines (data not shown). Therefore, it may be interesting to further investigate the role of mitochondrial dynamics in the development of cisplatin resistance in other adherent cancer cells with high transfection efficiency.

In conclusion, our data revealed that mitofusins Mfn1 and Mfn2 were upregulated in leukemia L1210 cells resistant to cisplatin. In addition, cisplatin stress increased the expression of mitofusins in parental L1210 cells. Inhibition of Drp1-dependent mitochondrial fission by Mdivi-1 efficiently attenuated cisplatin-induced cell death, intracellular ROS production and the activation of the caspase pathway in L1210 cells. These results demonstrate that mitochondrial dynamics play an important role in the antineoplastic effects of cisplatin in leukemia cells. Thus, targeting mitochondrial dynamics may provide a novel strategy in order to improve the chemotherapeutic effect of cisplatin in the future.

\section{Acknowledgements}

The present study was supported by the National Natural Science Foundation of China (81660607), the Natural Science Foundation of Jiangxi (20161 ACB20019), the Foundation of the Health and Family Planning Commission of Jiangxi Province (20161052), the Foundation of the Education Department of Jiangxi Province (150271), the Foundation of The Second Affiliated Hospital of Nanchang University (2014YNQN12012), and the Science Project of the Education Department of Hunan Province (16C0177).

\section{References}

1. Benton CB, Nazha A, Pemmaraju N and Garcia-Manero G: Chronic myelomonocytic leukemia: Forefront of the field in 2015. Crit Rev Oncol Hematol 95: 222-242, 2015.

2. Chavez-Gonzalez A, Bakhshinejad B, Pakravan K, Guzman ML and Babashah S: Novel strategies for targeting leukemia stem cells: sounding the death knell for blood cancer. Cell Oncol 40: $1-20,2017$.

3. Calado RT and Young NS: Telomere maintenance and human bone marrow failure. Blood 111: 4446-4455, 2008.

4. Safe S, Jin UH, Hedrick E, Reeder A and Lee SO: Minireview: Role of orphan nuclear receptors in cancer and potential as drug targets. Mol Endocrinol 28: 157-172, 2014.

5. Chen BA, Huang ZH, Zhang XP, Ou-Yang J, Li JY, Zhai YP, Sun XM, Xu YL, Lu Q, Wang JM, et al: An epidemiological investigation of leukemia incidence between 2003 and 2007 in Nanjing, China. J Hematol Oncol 3: 21, 2010.

6. Welch HG and Larson EB: Cost effectiveness of bone marrow transplantation in acute nonlymphocytic leukemia. N Engl J Med 321: 807-812, 1989.
7. Lenarsky $\mathrm{C}$ and Parkman R: Bone marrow transplantation for the treatment of immune deficiency states. Bone Marrow Transplant 6: 361-369, 1990.

8. Godder KT, Abhyankar SH, Lamb LS, Best RG, Geier SS, Pati AR, Gee AP and Henslee-Downey PJ: Donor leukocyte infusion for treatment of graft rejection post partially mismatched related donor bone marrow transplant. Bone Marrow Transplant 22: 111-113, 1998.

9. Kobayashi T, Yoshida J, Ishiyama J, Noda S, Kito A and Kida Y: Combination chemotherapy with cisplatin and etoposide for malignant intracranial germ-cell tumors. An experimental and clinical study. J Neurosurg 70: 676-681, 1989.

10. Borst P, Rottenberg S and Jonkers J: How do real tumors become resistant to cisplatin? Cell Cycle 7: 1353-1359, 2008.

11. Wong E and Giandomenico CM: Current status of platinum-based antitumor drugs. Chem Rev 99: 2451-2466, 1999.

12. Milacic V, Fregona D and Dou QP: Gold complexes as prospective metal-based anticancer drugs. Histol Histopathol 23: 101-108, 2008

13. Chen H, Hardy TM and Tollefsbol TO: Epigenomics of ovarian cancer and its chemoprevention. Front Genet 2: 67, 2011.

14. Wu Y, Bhattacharyya D, King CL, Baskerville-Abraham I, Huh SH, Boysen G, Swenberg JA, Temple B, Campbell SL and Chaney SG: Solution structures of a DNA dodecamer duplex with and without a cisplatin 1,2-d(GG) intrastrand cross-link: Comparison with the same DNA duplex containing an oxaliplatin 1,2-d(GG) intrastrand cross-link. Biochemistry 46: 6477-6487, 2007.

15. Podratz JL, Knight AM, Ta LE, Staff NP, Gass JM, Genelin K, Schlattau A, Lathroum L and Windebank AJ: Cisplatin induced mitochondrial DNA damage in dorsal root ganglion neurons. Neurobiol Dis 41: 661-668, 2011.

16. Oakes SA and Korsmeyer SJ: Untangling the web: Mitochondrial fission and apoptosis. Dev Cell 7: 460-462, 2004.

17. Szabadkai G, Simoni AM, Chami M, Wieckowski MR, Youle RJ and Rizzuto R: Drp-1-dependent division of the mitochondrial network blocks intraorganellar $\mathrm{Ca}^{2+}$ waves and protects against $\mathrm{Ca}^{2+}$-mediated apoptosis. Mol Cell 16: 59-68, 2004.

18. Shaw JM and Nunnari J: Mitochondrial dynamics and division in budding yeast. Trends Cell Biol 12: 178-184, 2002

19. Wan YY, Zhang JF, Yang ZJ, Jiang LP, Wei YF, Lai QN, Wang JB Xin HB and Han XJ: Involvement of Drp1 in hypoxia-induced migration of human glioblastoma U251 cells. Oncol Rep 32: 619-626, 2014

20. Chan DC: Mitochondrial fusion and fission in mammals. Annu Rev Cell Dev Biol 22: 79-99, 2006.

21. Santin G, Piccolini VM, Barni S, Veneroni P, Giansanti V, Dal Bo V, Bernocchi G and Bottone MG: Mitochondrial fusion: A mechanism of cisplatin-induced resistance in neuroblastoma cells? Neurotoxicology 34: 51-60, 2013.

22. Farrand L, Byun S, Kim JY, Im-Aram A, Lee J, Lim S, Lee KW, Suh JY, Lee HJ and Tsang BK: Piceatannol enhances cisplatin sensitivity in ovarian cancer via modulation of $\mathrm{p} 53$, X-linked inhibitor of apoptosis protein (XIAP), and mitochondrial fission. J Biol Chem 288: 23740-23750, 2013.

23. Kato S, Ideguchi $\mathrm{H}$, Muta $\mathrm{K}$, Nishimura $\mathrm{J}$ and Nawata $\mathrm{H}$ : Mechanisms involved in the development of adriamycin resistance in human leukemic cells. Leuk Res 14: 567-573, 1990.

24. Zwelling LA, Michaels S, Schwartz H, Dobson PP and Kohn KW: DNA cross-linking as an indicator of sensitivity and resistance of mouse L1210 leukemia to cis-diamminedichloroplatinum(II) and L-phenylalanine mustard. Cancer Res 41: 640-649, 1981.

25. Horvathova K, Novotny L and Vachalkova A: The free radical scavenging activity of four flavonoids determined by the comet assay. Neoplasma 50: 291-295, 2003.

26. Choi YM, Kim HK, Shim W, Anwar MA, Kwon JW, Kwon HK, Kim HJ, Jeong H, Kim HM, Hwang D, et al: Mechanism of cisplatin-induced cytotoxicity is correlated to impaired metabolism due to mitochondrial ROS generation. PLoS One 10: $\mathrm{e} 0135083,2015$.

27. Kaushal GP, Kaushal V, Hong X and Shah SV: Role and regulation of activation of caspases in cisplatin-induced injury to renal tubular epithelial cells. Kidney Int 60: 1726-1736, 2001.

28. Bai J, He A, Huang C, Yang J, Zhang W, Wang J, Yang Y, Zhang P, Zhang Y and Zhou F: Serum peptidome based biomarkers searching for monitoring minimal residual disease in adult acute lymphocytic leukemia. Proteome Sci 12: 49, 2014.

29. Muggia F: Platinum compounds 30 years after the introduction of cisplatin: Implications for the treatment of ovarian cancer. Gynecol Oncol 112: 275-281, 2009. 
30. Prestayko AW, D'Aoust JC, Issell BF and Crooke ST: Cisplatin (cis-diamminedichloroplatinum II). Cancer Treat Rev 6: 17-39, 1979.

31. Lebwohl D and Canetta R: Clinical development of platinum complexes in cancer therapy: An historical perspective and an update. Eur J Cancer 34: 1522-1534, 1998.

32. Galanski M: Recent developments in the field of anticancer platinum complexes. Recent Patents Anticancer Drug Discov 1: 285-295, 2006.

33. Chen $\mathrm{H}$ and Chan DC: Physiological functions of mitochondrial fusion. Ann NY Acad Sci 1201: 21-25, 2010.

34. Renault TT, Floros KV, Elkholi R, Corrigan KA, Kushnareva Y, Wieder SY, Lindtner C, Serasinghe MN, Asciolla JJ, Buettner C, et al: Mitochondrial shape governs BAX-induced membrane permeabilization and apoptosis. Mol Cell 57: 69-82, 2015.

35. Brooks C, Wei Q, Feng L, Dong G, Tao Y, Mei L, Xie ZJ and Dong Z: Bak regulates mitochondrial morphology and pathology during apoptosis by interacting with mitofusins. Proc Natl Acad Sci USA 104: 11649-11654, 2007.

36. Whelan RS, Konstantinidis K, Wei AC, Chen Y, Reyna DE, Jha S, Yang Y, Calvert JW, Lindsten T, Thompson CB, et al: Bax regulates primary necrosis through mitochondrial dynamics. Proc Natl Acad Sci USA 109: 6566-6571, 2012.
37. Wen S, Zhu D and Huang P: Targeting cancer cell mitochondria as a therapeutic approach. Future Med Chem 5: 53-67, 2013.

38. Tanaka A and Youle RJ: A chemical inhibitor of DRP1 uncouples mitochondrial fission and apoptosis. Mol Cell 29: 409-410, 2008.

39. Han XJ, Yang ZJ, Jiang LP, Wei YF, Liao MF, Qian Y, Li Y, Huang X, Wang JB, Xin HB, et al: Mitochondrial dynamics regulates hypoxia-induced migration and antineoplastic activity of cisplatin in breast cancer cells. Int J Oncol 46: 691-700, 2015.

40. Kim H, Lee JY, Park KJ, Kim WH and Roh GS: A mitochondrial division inhibitor, Mdivi-1, inhibits mitochondrial fragmentation and attenuates kainic acid-induced hippocampal cell death. BMC Neurosci 17: 33, 2016.

41. Akita M, Suzuki-Karasaki M, Fujiwara K, Nakagawa C, Soma M, Yoshida Y, Ochiai T, Tokuhashi Y and Suzuki-Karasaki Y: Mitochondrial division inhibitor-1 induces mitochondrial hyperfusion and sensitizes human cancer cells to TRAIL-induced apoptosis. Int J Oncol 45: 1901-1912, 2014.

42. Han XJ, Lu YF, Li SA, Kaitsuka T, Sato Y, Tomizawa K, Nairn AC, Takei K, Matsui $\mathrm{H}$ and Matsushita M: CaM kinase I alpha-induced phosphorylation of Drp1 regulates mitochondrial morphology. J Cell Biol 182: 573-585, 2008. 